\title{
IL-22: linking inflammation and cancer
}

Individuals with inflammatory bowel disease (IBD) have an increased risk of developing colon cancer, but there is a limited understanding of the immune cell types and cytokines that are involved in the transition from intestinal inflammation to cancer. A study published in the Journal of Experimental Medicine now shows that interleukin-22 (IL-22) is a key player in this transition.

First, the authors generated a new model of bacterial colitis-associated carcinoma in which mice that are genetically susceptible to colon cancer were infected with Helicobacter hepaticus and treated with the carcinogen 2-azoxymethane. These mice develop invasive colon cancer in 3-5 months. The authors found that these mice had increased numbers

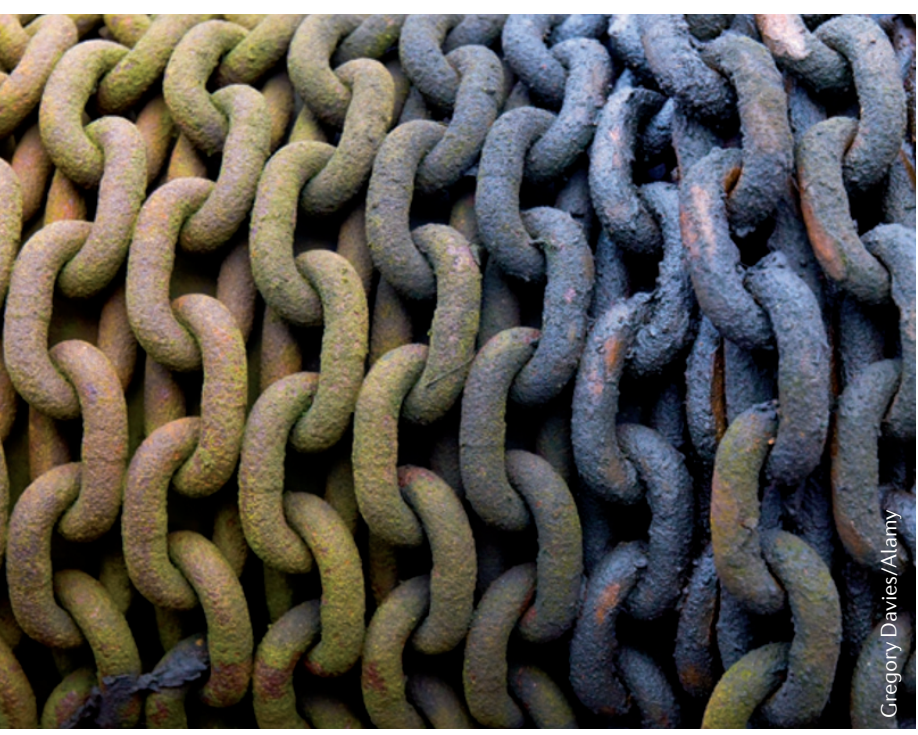

of innate lymphoid cells - immune cells that are involved in host defence and inflammation - and increased mRNA levels of several inflammatory cytokines in the colon. Treatment of these mice with an antibody that depleted innate lymphoid cells reduced inflammation and inflammatory cytokine levels in the colon and caecum, and also reduced the area of pre-carcinogenic aberrant crypt foci.

Further studies showed that the innate lymphoid cells from the mouse model produced IL-17 and IL-22, so the authors next investigated the effects of blocking these cytokines. Treatment with antibodies against IL-17 or IL-22 did not reduce the number of innate lymphoid cells, but it did reduce systemic and caecal inflammation. However, only IL-22 blockade ameliorated established colitis and reduced tumour burden, which suggests that IL-17 and IL-22 have distinct roles in inflammation and tumour development. As mice had developed tumours before IL-22 antibody treatment, these results suggest that IL-22 is involved in the maintenance of colon tumours as well as the transition from inflammation to cancer.

The authors hypothesized that IL-22 sustains inflammation and cancer through its actions on epithelial cells, leading to a dysregulated inflammatory response and tissue repair, which promotes cancer. Indeed, expression of the IL-22 receptor was restricted to colonic epithelial cells in the mouse model, and these cells had the phosphorylated form of the transcription factor STAT3 (signal transducter and activator of transcription 3) in their nuclei. Treatment of mice with an IL-22 antibody - but not IL-6 receptor or IL-17 antibodies reduced STAT3 phosphorylation, epithelial cell proliferation and the expression of other target genes of the IL-22-STAT3 axis. This suggests that IL-22 has a key role in regulating epithelial proliferation and antimicrobial defence through STAT3 phosphorylation.

Finally, the authors investigated IL-22 expression in tissue samples from individuals with sporadic colon cancer (samples from these patients were used because patients with IBD are screened to avoid cancer development). Analysis of IL-22 levels in matched pairs of human colon cancer tissue and adjacent tissue showed that IL22 mRNA expression in tumour tissue was more than twofold higher than in normal tissue in $58 \%$ of the pairs.

So this study shows that IL-22 produced by innate lymphoid cells provides a link between IBD and the development and maintenance of colon cancer, and suggests that targeting IL-22 might benefit some patients with colon cancer.

Charlotte Harrison

ORIGINAL RESEARCH PAPER Kirchberger, S. et al. Innate lymphoid cells sustain colon cancer through production of interleukin-22 in a mouse model.J. Exp. Med. 210, 917-931 (2013) 